Check for updates

Cite this: RSC Adv., 2018, 8, 29797

Received 20th June 2018

Accepted 10th August 2018

DOI: $10.1039 / c 8 r a 05295 f$

rsc.li/rsc-advances

\section{High yield production of cellulose by a Komagataeibacter rhaeticus PG2 strain isolated from pomegranate as a new host $\uparrow$}

\begin{abstract}
Meghana N. Thorat ${ }^{\mathrm{ab}}$ and Syed G. Dastager (D) *ab
Gluconacetobacter xylinus is a well-known organism that produces bacterial cellulose (BC). The present study was undertaken to find an alternative bacteria from a collection of 216 bacterial isolates, which were isolated from different rotten fruits and fermented beverages, to find a better producer of bacterial cellulose. We obtained a potent strain, which produced a high yield of BC from a rotten pomegranate sample, and was further identified as Komagataeibacter rhaeticus strain PG2 using 16S rRNA gene sequence analysis. To date, only two strains of Komagataeibacter rhaeticus are known to produce BC, and these were mainly isolated from a fermented beverage, kombucha. For the first time, we have isolated a BC producing Komagataeibacter rhaeticus strain PG2 from a rotten pomegranate sample. The new host environment and the substrate utilization pattern of strain PG2 reveal efficient bacterial cellulose production. Hestrin-Schramm (HS) liquid media containing glycerol as a carbon source resulted in the highest $B C$ production $\left(\sim 6.9 \mathrm{~g} \mathrm{~L}^{-1}\right)$. A further increased yield of $B C\left(\sim 8.7 \mathrm{~g} \mathrm{~L}^{-1}\right)$ was obtained by using $3 \%(\mathrm{w} / \mathrm{v})$ glycerol concentration, and this BC yield is the highest reported among any of the known Komagataeibacter rhaeticus strains reported. A detailed physico-chemical characterization of the BC membrane obtained from glycerol (Gly-BC) and glucose (Glc-BC) was performed. Interestingly, Gly-BC is found to be more compact and more crystalline in its nature compared to Glc$B C$. The present study reveals the isolation of an efficient BC synthesizing strain using glycerol as a lowcost carbon source, confirming the economic feasibility of BC production. The structural characteristics of the $\mathrm{BC}$ membrane produced by glycerol were found to be more suitable for various applications.
\end{abstract}

\section{Introduction}

Cellulose is the most abundant and sustainable biopolymer available on the Earth. Nanocellulose, with special morphology, a high surface area and unique mechanical properties like crystallinity, has attracted more attention for various biotechnological and biomedical applications., ${ }^{\mathbf{1 , 2}}$ Bacterial cellulose (BC), the nanofibrillar form of cellulose, has been exploited as a material for diverse applications due to its unique characteristics. Apart from bacterial cellulose, other exopolysaccharides were produced as extra polymeric materials which offer ecological fitness, and protect bacteria from environmental stress, UV radiation and toxic chemicals., ${ }^{2,3}$ Although BC is structurally similar to plant cellulose, its unique physicochemical characteristics, such as high tensile strength, a pure form (without having hemi-cellulose and lignin components), greater crystallinity and moldability make it superior to plant

${ }^{a}$ Academy of Scientific and Innovative Research (AcSIR), New Delhi, India. E-mail: sg. dastager@ncl.res.in

${ }^{b}$ NCIM Resource Center, CSIR-National Chemical Laboratory, Pune-411008, India

$\dagger$ Electronic supplementary information (ESI) available. See DOI: 10.1039/c8ra05295f cellulose. ${ }^{4-6}$ Among all of the types of nanocellulose, $\mathrm{BC}$ is naturally synthesized in the form of a 3D nanofibrillar network, bearing properties similar to those of collagen nanofibrils, and it possesses the compatibility required for biomedical applications. ${ }^{7}$ Properties like purity, biocompatibility, biodegradation and non-toxicity make BC a promising candidate for medical applications such as a bone and tissue scaffold material, a wound dressing material, a vascular graft and a drug delivery agent. ${ }^{5,8-10}$ Moreover, the wide range of intriguing properties of $\mathrm{BC}$ and its composites have paved the way for its various biotechnological applications like antimicrobials, biodegradable food packaging materials, emulsifiers, texture enhancers, flexure-durable paper, and as an additive in cosmetics., ${ }^{\mathbf{4 1 1}, \mathbf{1 2}}$

Komagataeibacter xylinus, previously called Gluconacetobacter xylinus, is the most exploited organism for BC production, and a number of references are available for $\mathrm{BC}$ production using this model organism. ${ }^{\mathbf{4} 13}$ However, there are very few reports of $\mathrm{BC}$ production using other strains of the Komagataeibacter genus, which may give better cellulose production with good structural properties. Despite having many superior properties compared to those of the plant counterpart, low yields and high production costs are the two major hurdles for industry in using this versatile polymer material for wider applications. Many 
attempts have been made to overcome these challenges by using different strains, improved bioprocessing techniques, cheaper carbon substrates and genetically modified organisms for better production of BC. ${ }^{\mathbf{1 4}}$ Recently Machado et al., used a $K$. rhaeticus strain obtained from traditional kombucha tea for the production of BC. ${ }^{15}$ The study claimed that the BC membrane obtained from $K$. rhaeticus had similar physicochemical properties to those obtained from G. xylinus, and therefore can be used as an alternative organism for cellulose production. A $K$. rhaeticus species was for the first time reported as a new alternative source for cellulose production from kombucha tea. ${ }^{16}$

In the present work, we report a cellulose producing bacterial strain, which is for the first time isolated from a rotten pomegranate sample, and identified it as $K$. rhaeticus by $16 \mathrm{~S}$ rRNA gene sequence analysis. The production of bacterial cellulose using different carbon sources was evaluated. The physicochemical characteristics of cellulose membranes obtained from different culture media were characterized by scanning electron microscopy (SEM), X-ray diffraction (XRD), attenuated total reflection Fourier transform infrared spectroscopy (ATR-FTIR) and $\mathrm{CP} / \mathrm{MAS}{ }^{13} \mathrm{C}$ nuclear magnetic resonance (NMR).

\section{Materials and methods}

\subsection{Isolation of cellulose producing bacteria}

Isolation from different rotten fruits was carried out, i.e., dragon fruit, mango, orange, sweet lime, citrus, banana, papaya, sapota, pear, carrot, apple, raisins, guava, fig, custard apple, musk melon, water melon, kiwi, star fruit, pineapple, dates and a fermented beverage (neera, a local drink also known as palm nectar; a sap extracted from the inflorescence of toddy palms). Samples were collected from a local market in Pune, India. Approximately $1 \mathrm{~g}$ of each of the fruit samples, and $1 \mathrm{~mL}$ of neera, were individually incubated in $25 \mathrm{~mL}$ of an enrichment medium (a modified Hestrin-Schramm (HS) media) containing (g L $\mathrm{L}^{-1}$ ): (glucose, 20; yeast extract, 5; peptone, 5; disodium hydrogen phosphate, 2.7; and citric acid, 1.15 at pH 6.0 supplemented with $100 \mathrm{ppm}$ of cycloheximide to inhibit the growth of yeasts and molds) at $28{ }^{\circ} \mathrm{C}$ for 10 days. ${ }^{17}$ The enriched samples were serially diluted and spread on glucose yeast extract (GYE) agar, and the plates were incubated at $28{ }^{\circ} \mathrm{C}$ for 4 days. Colonies which produced a clear zone of solubilization of $\mathrm{CaCO}_{3}$ on the agar medium were selected and purified. All isolates were purified by repeated-streaking on the HS agar medium, and the purified isolates were either preserved in $20 \%$ glycerol and stored at $-80{ }^{\circ} \mathrm{C}$, or re-streaked on HS agar slants which were stored at $4{ }^{\circ} \mathrm{C}$ until further use. A total of 216 bacterial isolates were further subjected to the bacterial cellulose production conditions using the HS liquid media containing $\left(\mathrm{g} \mathrm{L}^{-1)}\right.$ glucose, 20; yeast extract, 5; peptone, 5; disodium hydrogen phosphate, 2.7; citric acid, 1.15; $\mathrm{pH} 6.0^{18}$ and incubated for 10-15 d, to select the potential bacteria that are capable of BC production. The obtained BC was pre-treated as previously described. The formation of pellicles at the air-liquid interface was observed as a positive for BC producers. The pellicles formed are of cellulose material, or non-cellulosic, and this was confirmed by boiling them with $2 \% \mathrm{NaOH}$ for $30 \mathrm{~min}$
(ESI Fig. S1A and $\mathrm{B} \dagger$ ). The microfibrillar structures and crystallinity were observed using scanning electron microscopy (ESI Fig. S2A and $\mathrm{B} \dagger$ ).

\subsection{Identification of $\mathrm{BC}$ producing strains}

The potential BC producing bacterial isolates were cultivated in the HS liquid medium for 10 days, followed by centrifugation at $8000 \mathrm{~g}$ for $5 \mathrm{~min}$. The collected bacterial biomass was used to extract the total DNA. Isolation of genomic DNA was carried out by using a HiPurA ${ }^{\mathrm{TM}}$ (Hi-Media, Mumbai) DNA extraction kit according to the manufacturer's instructions. The 16S rRNA gene was amplified using universal primers $27 \mathrm{~F}$ (5'-AGAGTTTGATCCTGGCTCAG- $3^{\prime}$ ) and 1492R (5'-CGGTTACCTTGTTACGACTT- $3^{\prime}$ ). The PCR conditions used for amplification of the 16S gene were as follows: an initial denaturation at $94{ }^{\circ} \mathrm{C}$ for $3 \mathrm{~min}$, an elongation of 35 cycles at $94{ }^{\circ} \mathrm{C}$ for $30 \mathrm{~s}$, followed by $55{ }^{\circ} \mathrm{C}$ for $30 \mathrm{~min}$ and $72{ }^{\circ} \mathrm{C}$ for $1 \mathrm{~min} 30 \mathrm{~s}$, and a final elongation at $72{ }^{\circ} \mathrm{C}$ for $7 \mathrm{~min}$. The PCR product was purified using an ExoSAP (New England Bio labs) and sequenced using a 3500XL DX Genetic Analyzer (Applied Biosystems, USA). The purified PCR fragments were used for sequencing in the 3500XL DX Genetic Analyzer (Applied Biosystems, USA). The nearest neighbours of the strains were determined by comparing the 16S rRNA gene sequences with available sequences in the NCBI GenBank using the BLAST program. A phylogenetic tree was constructed after multiple alignments of the sequences of the most closely related strains retrieved from the Ezbiocloud database (http:// www.ezbiocloud.net). A neighbour joining tree method was used from MEGA (version 7.0) to find the positions of the BC producing isolates in the phylogenetic tree. The topology of the phylogenetic tree was evaluated with bootstrap values based on 1000 replications.

\subsection{Effect of carbon sources on bacterial cellulose production}

Potential BC producing strains were further evaluated to investigate the effect of different carbon sources on their BC production. Glucose from the standard HS media was replaced with different sugars and sugar alcohols. Fructose, lactose, xylose, sucrose, galactose, mannitol, sorbitol and glycerol were used as the carbon sources, one at a time, in the given HS liquid medium by replacing the glucose. Based on the carbon source analysis, the source which tended to give the highest BC yield was further subjected to studies investigating the effect of different $\mathrm{pH}$ (3.0 to 7.0) on BC production. All of the representative graphs of analysis were plotted using GraphPad Prism (GraphPad Software Inc.). To investigate the carbon source efficiency on the BC-production yield, one loop of a celluloseproducing isolate was transferred to $100 \mathrm{~mL}$ of the HS liquid medium in a $250 \mathrm{~mL}$ flask, and incubated at $28{ }^{\circ} \mathrm{C}$ for $72 \mathrm{~h}$ as a starter culture. Five percent $(\mathrm{v} / \mathrm{v})$ of the starter culture was then transferred to $100 \mathrm{~mL}$ of the HS medium in a $250 \mathrm{~mL}$ flask, and incubated at $28^{\circ} \mathrm{C}$ for $10-15$ days. The pellicle formed at the airliquid interface was collected and rinsed with water 2 to 3 times to remove bacterial debris. After the water wash, the $\mathrm{BC}$ membrane was treated with $2 \% \mathrm{NaOH}$ at $80{ }^{\circ} \mathrm{C}$ for $30 \mathrm{~min}$, 
followed by repeated washing with distilled water until neutral $\mathrm{pH}$ was obtained. The resulting cellulose membrane is free from microbial cells and media components. The purified pellicle obtained was lyophilized and weighed to determine its dry weight $\left(\mathrm{g} \mathrm{L}^{-1}\right)$.

\subsection{Characterization of bacterial cellulose}

2.4.1. X-ray diffractometry (XRD). The X-ray diffraction patterns of the lyophilized BC samples were recorded by powder XRD on a PANalytical X'Pert PRO powder X-ray diffractometer with $\mathrm{Cu} \mathrm{K} \alpha$ wavelength $(\lambda=0.1541 \mathrm{~nm})$ operating at tension 40 $\mathrm{kV}$ and current $30 \mathrm{~mA}$. The diffractogram was obtained from $2 \theta$ angles ranging from $10^{\circ}$ to $80^{\circ}$. The Crystallinity Index (Cr I) was determined using the Segal equation as mentioned below. ${ }^{15,19}$

$$
\mathrm{Cr} \mathrm{I}=\frac{I_{002}-I_{\mathrm{am}}}{I_{002}} \times 100
$$

where $I_{002}$ is the intensity of the peak at $2 \theta \approx 22.5$, and $I_{\mathrm{am}}$ is the intensity at $2 \theta \approx 18^{\circ}$.

2.4.2. Field emission scanning electron microscopy (FESEM). For scanning electron microscopy (SEM) analysis, a small piece of the lyophilized $\mathrm{BC}$ membrane was coated with gold using a sputter coater. The fibrillar morphology of the specimens was observed with a FEI Quanta FEG 450 scanning electron microscope operating at $10 \mathrm{kV}$.

2.4.3. Attenuated total reflection-Fourier transform infrared spectroscopy (ATR-FTIR). FTIR spectra of the lyophilized BC samples were recorded on a Spectrum Two Perkin Elmer instrument. The samples were analyzed using the ATR mode on a diamond ATR accessory. The FTIR spectra were recorded in the range of $4000-400 \mathrm{~cm}^{-1}$ wavenumbers with a resolution of $4 \mathrm{~cm}^{-1}$ and an accumulation of 32 scans.

2.4.4. ${ }^{13} \mathrm{C}$ (CPMAS) nuclear magnetic resonance (NMR). Solid-state NMR was carried out on ${ }^{13} \mathrm{C}$ with a frequency of 100.5 MHz on a $400 \mathrm{MHz}$ NMR spectrometer. ${ }^{13} \mathrm{C}$ (CPMAS) cross-polarization spectra were measured with a magic angle spinning at $8 \mathrm{kHz} ; \pi / 2$ pulse, $1 \mathrm{~ms}$. The spectra were referenced against adamantane.

2.4.5. Thermogravimetric analysis (TGA). The thermogravimetric curves of the lyophilized BC membrane were recorded by an SDT model Q 600 of TA Instruments Inc. USA. All samples were heated in alumina pans from a $30{ }^{\circ} \mathrm{C}$ to $800{ }^{\circ} \mathrm{C}$ temperature range under a nitrogen atmosphere (flow rate of $20.0 \mathrm{~mL} \mathrm{~min}^{-1}$ ) at a heating rate of $10{ }^{\circ} \mathrm{C} \min ^{-1}$.

\section{Results and discussion}

\subsection{Isolation and characterization of $\mathrm{BC}$ producing bacteria}

A total of 216 bacterial isolates were isolated from different rotten fruits and the fermented liquid neera that were collected from a local market in Pune. All of the isolates were primarily examined for acid production on solid media using calcium carbonate as a substrate, since $\mathrm{BC}$ production has a direct relationship with acid production. A total of six isolates, two from pomegranate (PG1, PG2), two from dragon fruit (DF 10, DF 18) and one each from mango (Man 5) and neera (N10) were found positive for acid production, which showed a clear zone around the colonies (ESI Fig. S1 $\dagger$ ). These were preserved and further investigated for BC production using standard the HS liquid medium. All six of the strains were found positive for BC production in the liquid HS medium, and were further identified using 16S rRNA gene sequencing. The sequences were deposited in the NCBI GenBank with the following accession numbers; MH482915 (1382bp), MH484047 (1454), MH484163 (1315bp), MH482946 (1460bp), MH484052 (1460bp) and MH484172 (1467bp). Phylogenetic analysis was done by retrieving the sequences from the NCBI GenBank database for further analysis. Phylogenetically, all six of the BC producing isolates were clubbed into three groups of the previously reported Komagataeibacter genus, i.e., strain PG2 as K. rhaeticus, strain N-10 as $K$. intermedius and the DF18, Man5, DF10 and PG1 strains as $K$. hansenii with a sequence similarity ranging from 99.84, 100, 99.45, 99.86, 99.86 and 99.86\% respectively (Fig. 1), since the all of the isolates share a higher sequence similarity above $98.6 \%$, and are a threshold value for any novel species description. Since Komagataeibacter is re-grouped from the genus Gluconacetobacter, a typical and widely-studied BCproducing family, the potential strain $K$. rhaeticus PG-2, isolated in this study showed a good yield of BC, and could be a good candidate for BC preparation.

\subsection{Bacterial cellulose production from $K$. rhaeticus strain PG2}

Among the six isolates, the $K$. rhaeticus strain PG2 was found to have more potential, as it showed the highest $\mathrm{BC}$ production $\left(\sim 4.0 \mathrm{~g} \mathrm{~L} \mathrm{~L}^{-1}\right)$ in the standard HS liquid medium. The $\mathrm{BC}$ produced was in the form of a thick gelatinous membrane during static cultivation, where the organisms remained entrapped in the fibrillar network (Fig. 2). The BC production ( $\mathrm{g} \mathrm{L}^{-1}$ ) by the $K$. rhaeticus strain PG2 was further assessed using different sugars and sugar alcohols (Fig. 3a) varying one at a time as the carbon source. It was observed that the $\mathrm{BC}$ production from glycerol was recorded as the highest ( $\left.\sim 6.9 \mathrm{~g} \mathrm{~L}^{-1}\right)$, among the other carbon sources tested, followed by glucose $\left(\sim 4.05 \mathrm{~g} \mathrm{~L}^{-1}\right)$. The sugar alcohols like sorbitol and mannitol were found to enhance $\mathrm{BC}$ production in the range of $\sim 1.65 \mathrm{~g} \mathrm{~L}^{-1}$ to $\sim 3.41 \mathrm{~g} \mathrm{~L}^{-1}$ respectively. The fructose-rich media produced $\sim 2.7 \mathrm{~g} \mathrm{~L}^{-1}$ of BC. The sugars like maltose, mannose, lactose, xylose, sucrose and galactose appeared to give poor $\mathrm{BC}$ yields, and were found to be less efficient carbon sources for BC production by the $K$. rhaeticus strain PG2.

The low yield of BC when sucrose was used as a carbon source may be due to the inability of the organism to transport sucrose inside cells. ${ }^{20}$ According to Ishihara et al. ${ }^{21}$ a low yield of $\mathrm{BC}$ was reported when xylose was used as a carbon source, and this is attributed to the accumulation of xylonic acid in the media, which hampers the cell growth and hence explains the low yield of BC production. ${ }^{21,22}$ Similarly, the organism cannot utilize lactose, which may be due to the absence of the enzyme $\beta$-glucosidase mechanism which is required for the breakdown of lactose. ${ }^{22}$ Overall, glycerol gave the highest production of BC $\left(\sim 6.9 \mathrm{~g} \mathrm{~L}^{-1}\right)$ compared to the other carbon sources without 


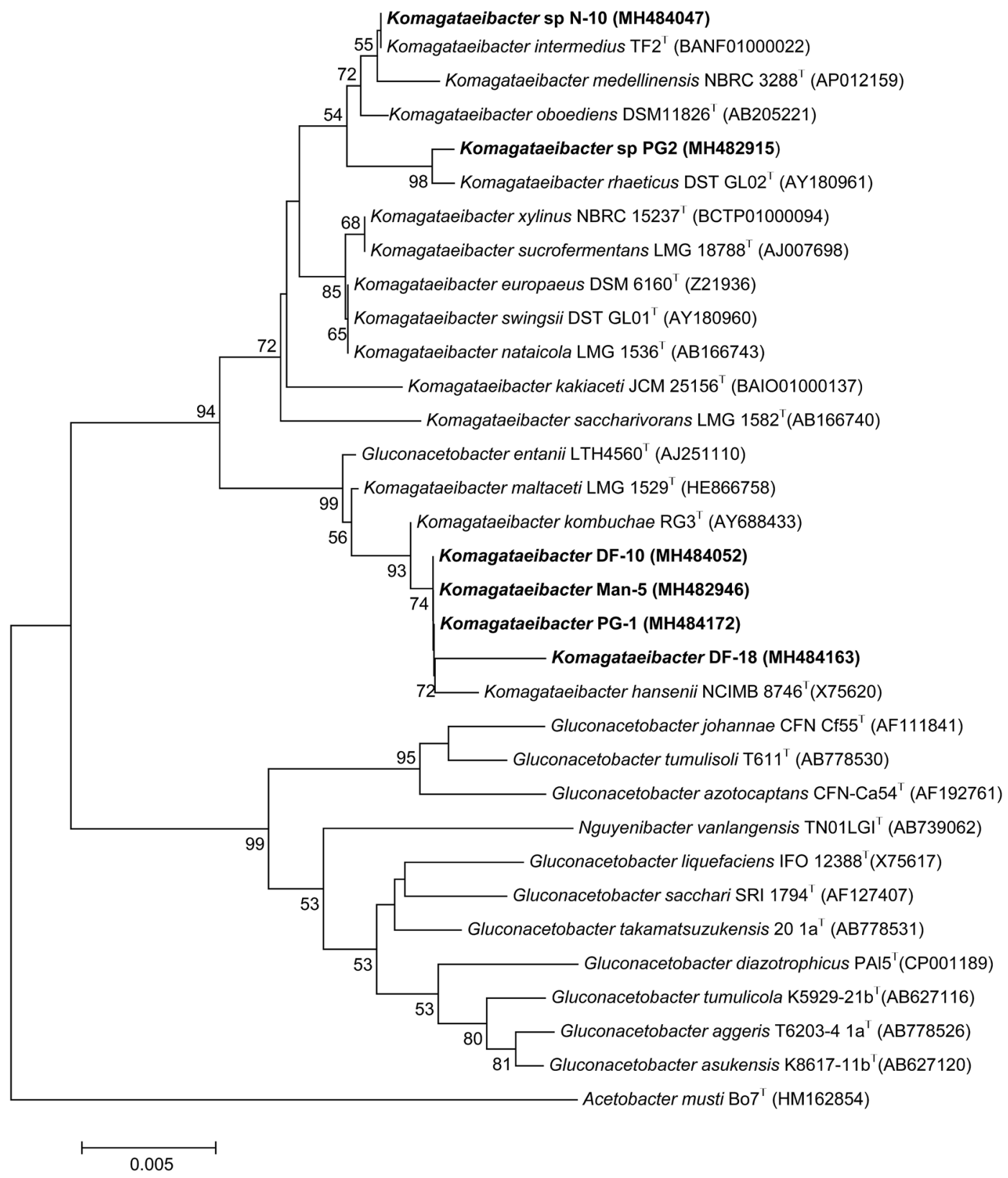

Fig. 1 A neighbour joining phylogenetic tree showing the relationships between the BC-producing isolates and their closest neighbours. The numerals at the branching points indicate the bootstrap values (\%) obtained after 1000 replications. Acetobacter musti Bo7 (HM162854) served as an outgroup.
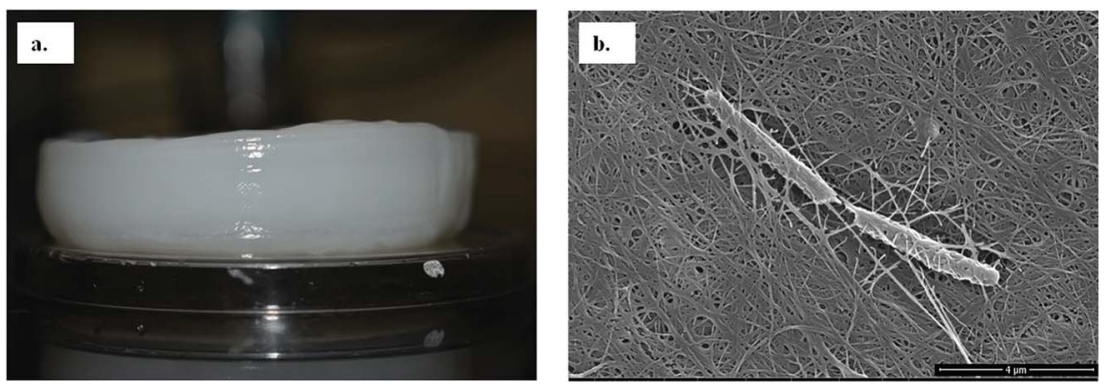

Fig. 2 (a) The pure wet BC membrane produced by the K. rhaeticus strain PG2 using glycerol as the carbon source after 15 days of incubation at $28{ }^{\circ} \mathrm{C}$ under static cultivation. (b) A FE-SEM image of the bacterial cellulose membrane network with the entrapped $K$. rhaeticus strain PG2. 

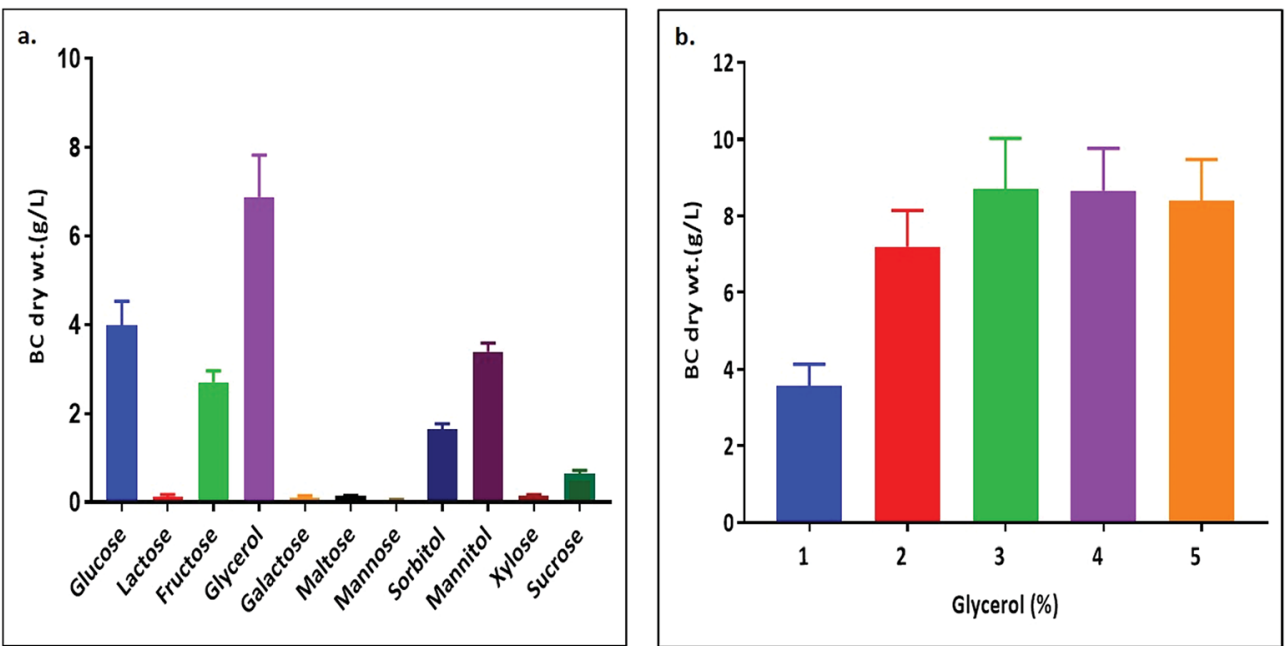

Fig. $3 \mathrm{BC}$ membrane production by the $K$. rhaeticus strain $\mathrm{PG} 2\left(\mathrm{~g} \mathrm{~L}^{-1}\right)(\mathrm{a})$ from different carbon sources, and (b) with different glycerol \% (w/v) after 15 days of incubation at $28^{\circ} \mathrm{C}$ under static cultivation.

a change in the initial $\mathrm{pH}$ value of $\sim 5.7$, while others have drastically changed the $\mathrm{pH}$ value up to 3.0. It is known that glycerol switches its path from the pentose cycle to the Krebs cycle without accumulation of gluconic acid. Oppositely, in the case of glucose, the membrane-bound dehydrogenases convert the glucose into a by-product such as gluconic acid, which results in the decline of $\mathrm{pH}(\sim 3.4))^{20,23}$ Low $\mathrm{pH}$ values and accumulation of the by-product gluconic acid could be one of the main reasons for the relatively low yield of cellulose production when glucose was used as the sole carbon source. ${ }^{20}$ It is a universal phenomenon that the pattern of utilization of carbon sources varies from strain to strain. In the present study we have demonstrated that the $K$. rhaeticus strain PG2 can utilize glycerol, glucose, mannitol, sorbitol and fructose efficiently for $\mathrm{BC}$ production. We also analysed the effect of different glycerol concentrations $(1 \%, 2 \%, 3 \%, 4 \%$, and $5 \% \mathrm{w} / \mathrm{v})$ on the $\mathrm{BC}$ production from the K. rhaeticus, strain PG2, and a significant enhancement in the $\mathrm{BC}$ yield was observed from $7.1 \pm 0.9$ to $8.7 \pm 1.3 \mathrm{~g} \mathrm{~L}^{-1}$ when the glycerol concentration was increased from $2 \%$ to $3 \%$ (Fig. $3 \mathrm{~b}$ ). The previous reports for the highest BC production using strains of $K$. rhaeticus were only reported using glucose as the carbon source, where the authors have used $2 \%$ and $5 \%$ glucose as the carbon source and obtained $\mathrm{BC}$ yields in the range of $\sim 4.77$ to $\sim 6.7 \mathrm{~g} \mathrm{~L}^{-1}$ by the $K$. rhaeticus strain $\mathrm{P} 1463$, and the $K$. rhaeticus strain AF-1 respectively. ${ }^{15,24}$ In the present study, we have demonstrated the highest yield of $\mathrm{BC}$ production $\left(8.7 \mathrm{~g} \mathrm{~L}^{-1}\right)$ using $3 \%$ glycerol as the carbon source, which is relatively cheaper and a more readily available substrate compared to glucose, thereby converting glycerol into the value added nanocellulose (BC) is a more promising approach for economical production.

Previous reports suggest that utilization of different carbon sources, as well as the optimum $\mathrm{pH}$ for $\mathrm{BC}$ production, is dependent on the kind of bacteria used for the $\mathrm{BC}$ synthesis. The optimum $\mathrm{pH}$ for $\mathrm{BC}$ production has usually been reported to be within a range from neutral to slightly acidic. ${ }^{13}$ We have tested the $\mathrm{pH}$ in the range of acidic to neutral $(3.0-7.0)$ for its effect on BC production by using the $K$. rhaeticus strain PG2 in the HS liquid medium with glycerol as the sole carbon source (Fig. 4). The maximum yields of cellulose production were observed at pH $5.0\left(\sim 7.16 \mathrm{~g} \mathrm{~L}^{-1}\right)$ and $6.0\left(\sim 7.15 \mathrm{~g} \mathrm{~L}^{-1}\right)$ respectively. The $\mathrm{BC}$ production slows down when $\mathrm{pH}$ was decreased. The lowest production of $\mathrm{BC}$ was observed at $\mathrm{pH} 7.0\left(2.39 \mathrm{~g} \mathrm{~L}^{-1}\right)$. The decreased yield of $\mathrm{BC}$ produced at a lower $\mathrm{pH}$ is due to the inhibition of bacterial growth. It was also observed that the slightly lower acidic environment is favourable for acetic acid bacteria, which justifies the higher yield of $\mathrm{BC}$ production at $\mathrm{pH}$ 5.0 and $6.0 .^{25}$ Thus the strain $K$. rhaeticus $\mathrm{PG} 2$ follows a similar pattern of $\mathrm{BC}$ production in different $\mathrm{pH}$ as reported earlier. ${ }^{25}$

Many researchers have demonstrated that the production of $\mathrm{BC}$, as well as the physicochemical properties, are influenced by several factors which include cultural conditions $(\mathrm{pH}$, temperature, and incubation time), media components (carbon source and nitrogen source), and are further followed by the kind of bacterial strains used for the production. ${ }^{22}$ All of these variables

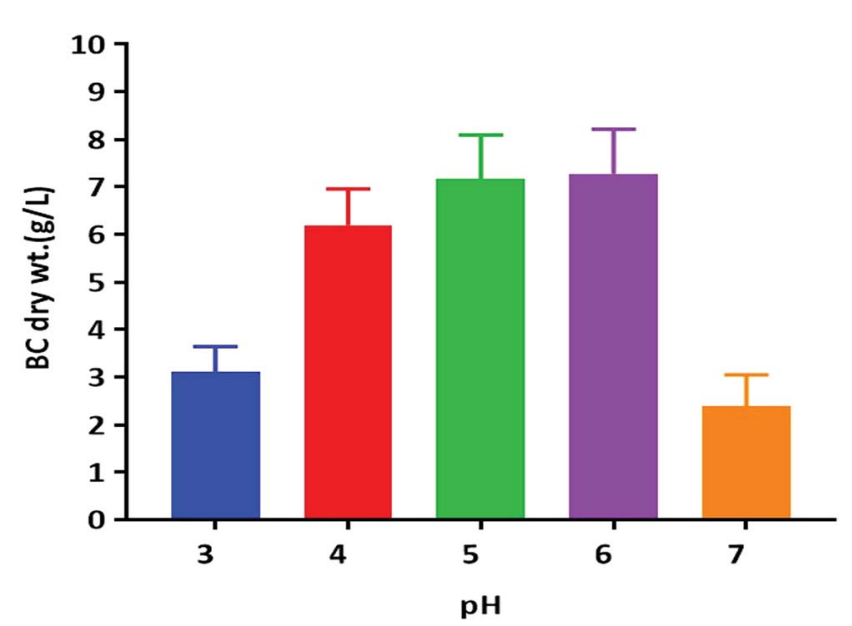

Fig. 4 BC membrane production by the $K$. rhaeticus strain PG2 $\left(\mathrm{g} \mathrm{L}^{-1}\right)$ at various $\mathrm{pH}$ levels after 15 days of incubation at $28^{\circ} \mathrm{C}$ under static cultivation. 
have a considerable impact on the material properties. The different applications need a material with varied properties. Therefore, in-depth understanding of the material properties are important.

\subsection{Characterization of bacterial cellulose}

The X-ray diffractogram (XRD) for bacterial cellulose samples were obtained from BC producing isolates and compared with K. xylinus LMG $1518^{\mathrm{T}}$. The XRD patterns of the most suitable carbon sources for the strain $K$. rhaeticus PG2 are given in Fig. 5A and B. The XRD patterns obtained are very similar to the pattern of cellulose I, showing the characteristics peaks at $2 \theta$ angles $\approx 14^{\circ}, 16^{\circ}$ and $22^{\circ}$, and the absence of peaks at $2 \theta$ angles $\approx 12^{\circ}, 20^{\circ}$ which is the typical pattern of cellulose II. $^{26}$ The crystallinity index (Cr I) of the BC membranes obtained using different carbon sources from the $K$. rhaeticus strain PG2 was calculated using the Segal method (peak height) and is presented in Table 1 . The highest crystallinity index was recorded for the $\mathrm{BC}$ obtained from glycerol (i.e., 80.79) whereas the lowest $\mathrm{Cr}$ I was obtained for the BC membranes from the fructose $(\sim 58.14)$ and sorbitol $(\sim 58.91)$ sources respectively. The Cr I of the BC membranes obtained from glucose and mannitol were $\sim 75.35$ and $\sim 76.38$ respectively.

The detailed physicochemical characterizations of the $\mathrm{BC}$ membranes obtained from glycerol (Gly-BC) and glucose (Glc$\mathrm{BC}$ ) were further analyzed owing to their high production. Scanning electron microscope images of lyophilized Glc-BC and Gly-BC membrane are presented in Fig. 6a and b. SEM analysis revealed that a more compact network of $\mathrm{BC}$ membranes were obtained from the glycerol source, whereas a porous and less compact reticular network structure of $\mathrm{BC}$ was observed from the glucose source. There are no significant differences in the sizes of the microfibril widths of the BC membranes obtained from both of the carbon sources. Both of the sources have a fibrillar width in the range of $30-80 \mathrm{~nm}$. Fig. $6 \mathrm{c}$, $\mathrm{d}$ and $\mathrm{f}$ provide the transverse-sectional insights of the $\mathrm{BC}$ membrane. The transverse section of Gly-BC showed well-organized pores, and most of them were ovoid in shape, whereas Glc-BC showed a less organized porous network of microfibrils. Fig. $6 \mathrm{f}$ indicates
Table 1 Crystallinity indexes calculated using the Segal equation for the most suitable carbon sources compared with the $K$. xylinus LMG $1518^{\top}$ grown in the standard HS medium

\section{Carbon source}

Crystallinity index(Cr I)

Glycerol

Mannitol

80.80

Glucose

76.40

Sorbitol

75.35

Fructose

58.91

K. xylinus LMG $1518^{\mathrm{T}}$ (std HS medium)

that the channels are interconnected with short fibrils, in which the arrows indicate the alignment of the pores in the membrane.

Further, the CP/MAS ${ }^{13} \mathrm{C}$ NMR spectra for Gly-BC and Glc-BC are shown in Fig. 7. All of the resonance lines were assigned for C-1 to C-6. ${ }^{27}$ Both of the spectra are very similar and show resonance lines at 102-108 ppm, 81-93 ppm and 60-68 ppm which were assigned to the $\mathrm{C}-1, \mathrm{C}-4$ and $\mathrm{C}-6$ carbons respectively. The clusters of resonances from $70 \mathrm{ppm}$ to $80 \mathrm{ppm}$ were assigned to the C-2, C-3 and C-5 carbons. ${ }^{27,28}$ It is known that cellulose $\mathrm{I}$ is composed of two distinct crystalline structures i.e., $I_{\alpha}$ and $I_{\beta}$. The fractions of both of the allomorphs vary depending on the source of cellulose. Mostly, cellulose from bacteria and valonia is $I_{\alpha}$ rich, whereas plant-based cellulose is rich in the $\mathrm{I}_{\beta}$ crystalline phase. ${ }^{29}$ Due to the presence of smaller doublets beside the prominent central resonance line, $\mathrm{C}-1$ and C-4 appear to be triplets, indicating the presence of a minor fraction of the $I_{\beta}$ phase and a major fraction of the $I_{\alpha}$ phase of cellulose. $^{13,29}$

Further structural analyses of the Gly-BC and Glc-BC membranes were carried out by FTIR analysis. The FTIR spectra obtained for the Gly-BC and Glc-BC membranes are in the range of $4000-500 \mathrm{~cm}^{-1}$, and are represented in Fig. 8a. Both of the spectra obtained showed the presence of characteristic bands known for cellulose I. The sharp peaks at position $3344 \mathrm{~cm}^{-1}$ of $(\mathrm{O}(3) \mathrm{H} \cdots \mathrm{O}(5))$ and $3242 \mathrm{~cm}^{-1}$ show the
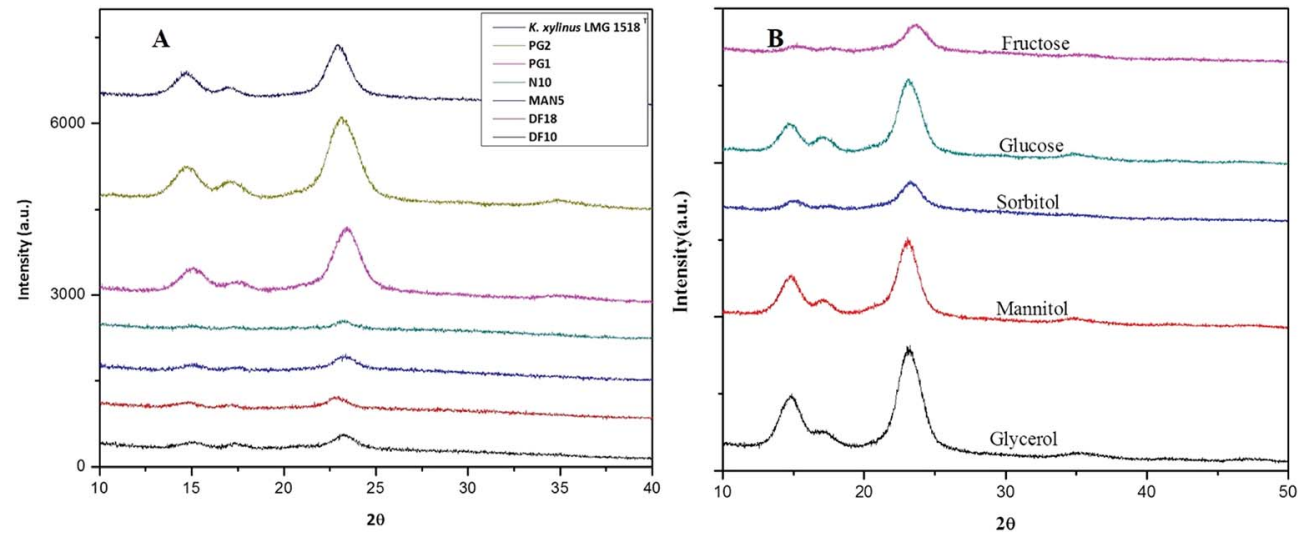

Fig. 5 XRD spectra of BC membranes produced (A) by all of the isolates when compared to $K$. xylinus $\mathrm{LMG}^{1518^{\top}}$, and (B) by the $K$. rhaeticus strain PG2 under different carbon conditions after 15 days of incubation at $28{ }^{\circ} \mathrm{C}$ under static cultivation. 

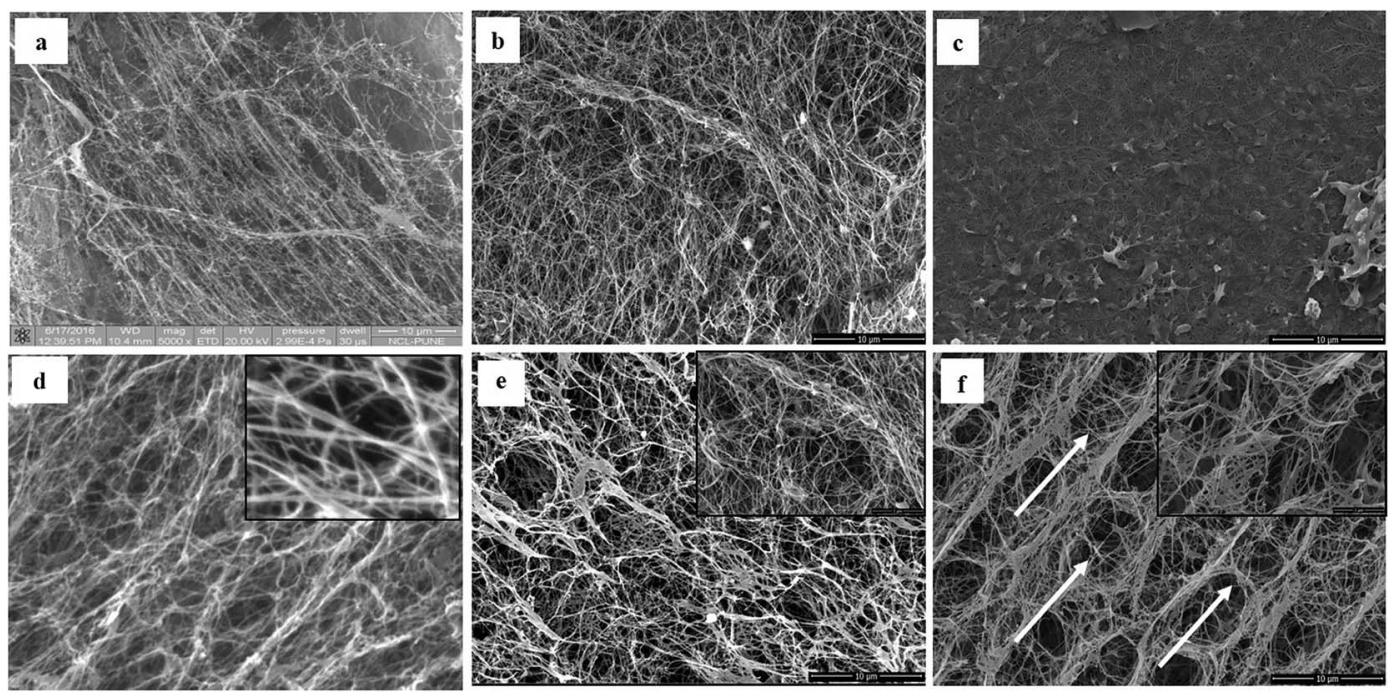

Fig. 6 Scanning electron micrograph images showing a comparison of the pattern of the bacterial cellulose membrane produced by $K$. xylinus LMG $1518^{\top}$, and the $K$. rhaeticus strain PG2 from the HS with glucose, and from the HS with glycerol, after 15 days of incubation at $28^{\circ} \mathrm{C}$ under static cultivation. Surface view of (a) K. xylinus LMG 1518 ${ }^{\top}$, (b) the K. rhaeticus strain PG2 (Glc-BC) and (c) the K. rhaeticus strain PG2 (Gly-BC). The transverse sections of the same membranes are mentioned in $(d-f)$. The arrows in ( $f$ ) indicate the uniform hexagonal arrangement of the membrane fibrillar structures.

intermolecular hydrogen bonding of $(\mathrm{O}(6) \mathrm{H} \cdots \mathrm{O}(3))$ which are typical peaks of $-\mathrm{OH}$ stretching vibrations, indicating the properties of cellulose I. Absence of bands in the regions of $3488 \mathrm{~cm}^{-1}$ and $3447 \mathrm{~cm}^{-1}$ (OH stretching due to intramolecular hydrogen bonding) confirms the absence of the cellulose II properties. ${ }^{15,30}$ The signature peak for cellulose I at $1430 \mathrm{~cm}^{-1}$ (a symmetric $\mathrm{CH}_{2}$ bending vibration) further confirms the property cellulose $\mathrm{I}$ in the structure. ${ }^{30}$ The complex fingerprint regions ranging from $1800-800 \mathrm{~cm}^{-1}$ showed the following characteristic bands; $1651 \mathrm{~cm}^{-1}$ (water absorbed), $1427 \mathrm{~cm}^{-1}$ ( $\mathrm{CH}_{2}$ symmetric bending), $1370 \mathrm{~cm}^{-1}$ ( $\mathrm{CH}$ bending), $1337 \mathrm{~cm}^{-1}$ (OH in-plane bending), $1315 \mathrm{~cm}^{-1}\left(\mathrm{CH}_{2}\right.$ wagging), $1280 \mathrm{~cm}^{-1}$
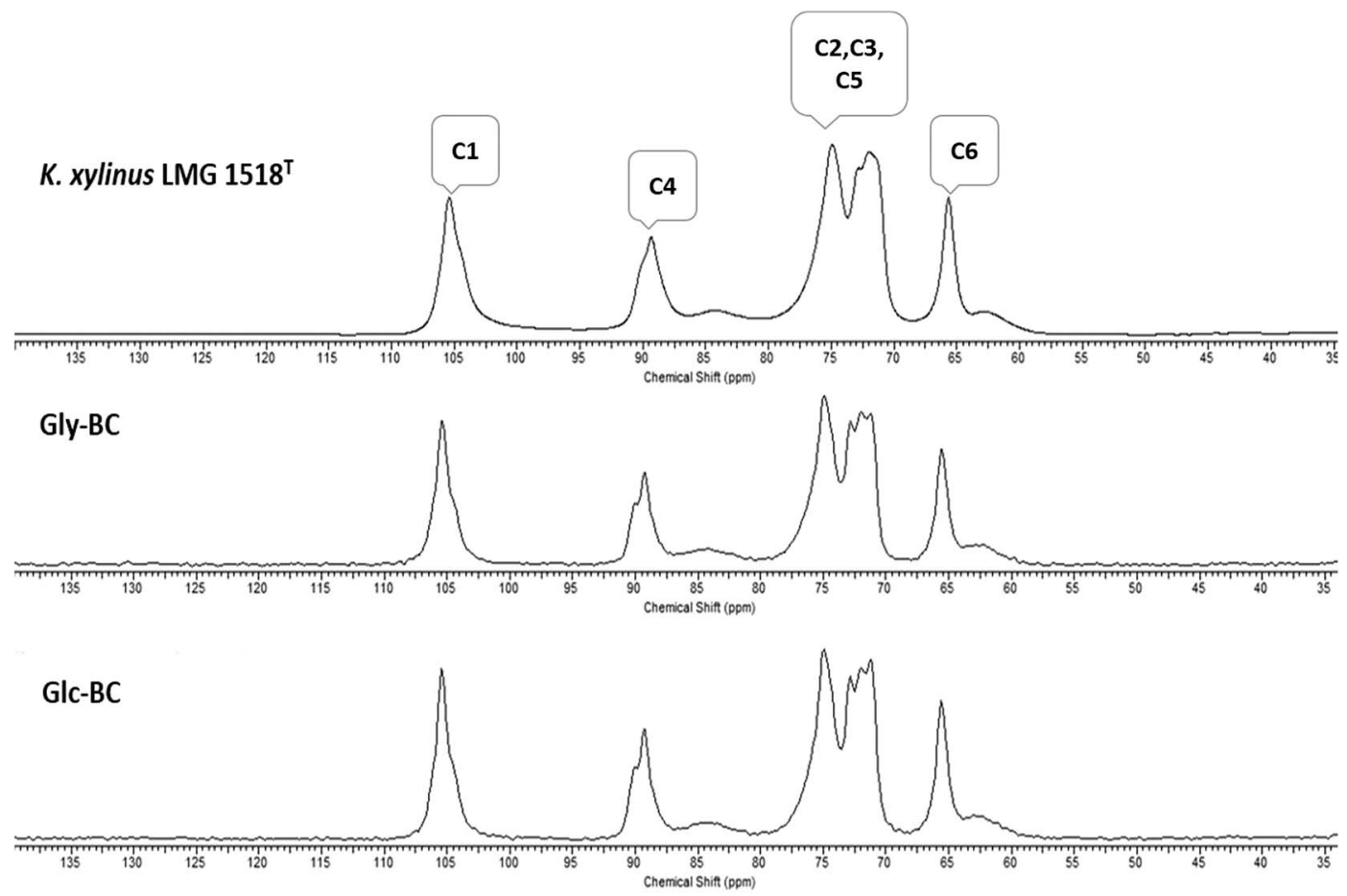

Fig. 7 Comparison of CP/MAS ${ }^{13} \mathrm{C}$ NMR spectra for the $\mathrm{BC}$ membrane obtained from $K . x y l i n u s$ LMG $1518^{\top}$ in the standard HS-medium with the K. rhaeticus strain PG2 obtained from the standard HS (Glc-BC), and modified HS with glycerol (Gly-BC), after 15 days of incubation at $28{ }^{\circ} \mathrm{C}$ under static cultivation. 

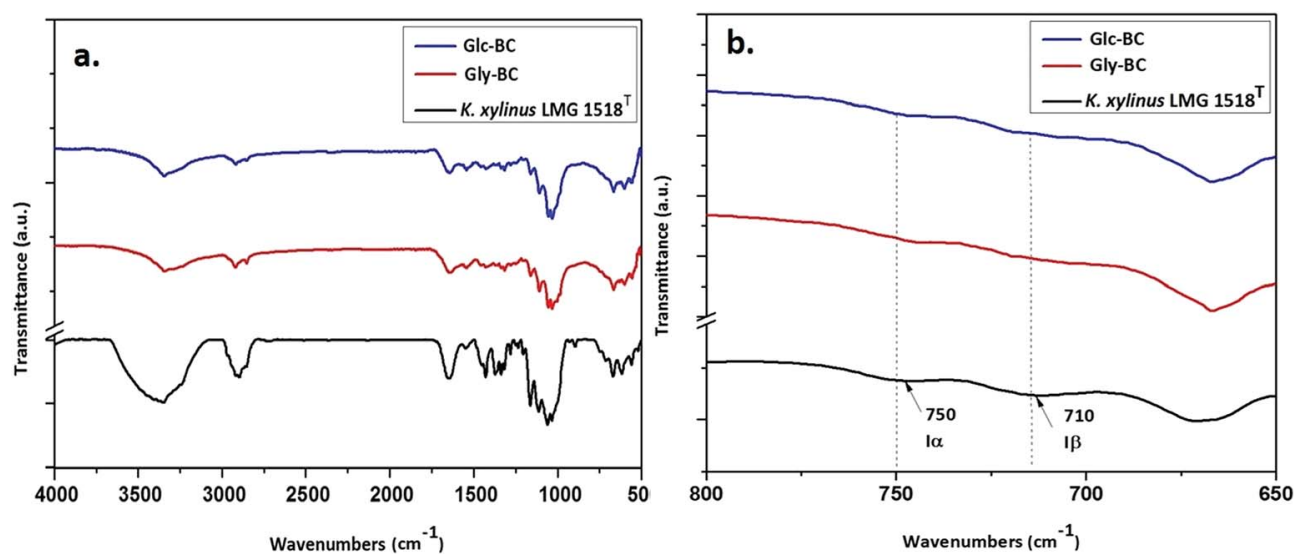

Fig. 8 (a) FTIR spectra comparison of the BC membrane produced by $K$. xylinus LMG $1518^{\top}$ grown in the standard HS-medium with the $K$. rhaeticus strain PG2 grown in the standard HS medium (Glc-BC), and the modified HS medium with glycerol (Gly-BC); (b) FTIR spectra in the range of $800-650 \mathrm{~cm}^{-1}$ indicating the $I_{\alpha}$ and $I_{\beta}$ allomorphs.

(CH bending), $1161 \mathrm{~cm}^{-1}$ (C-O-C asymmetric stretching at the $\beta$-glucosidic linkage), $1108 \mathrm{~cm}^{-1}(\mathrm{C}-\mathrm{O}$ bond stretching), $1054 \mathrm{~cm}^{-1}$ (C-O-C ring skeletal vibration), and $896 \mathrm{~cm}^{-1}$ (weak and broad bands of a $\beta$-linked polymer). ${ }^{28,31,32}$ The given FTIR spectra reveal the existence of both the $\mathrm{I}_{\alpha}$ and $\mathrm{I}_{\beta}$ allomorphs in Gly-BC and Glc-BC, which are in agreement with the NMR results obtained in the present study. The peaks at $3220 \mathrm{~cm}^{-1}$ and $750 \mathrm{~cm}^{-1}$, and $3283 \mathrm{~cm}^{-1}$ and $710 \mathrm{~cm}^{-1}$, have been assigned to the triclinic allomorph $\mathrm{I}_{\alpha}$ and the monoclinic allomorph $\mathrm{I}_{\beta}$ respectively. ${ }^{26,28}$

The thermal degradation behaviour patterns of the Gly-BC and Glc-BC membranes are provided in Fig. 9. Both curves showed the same thermal properties with two main weight losses. The initial minor weight loss curve observed was associated with the evaporation of surface water. At a temperature around $270{ }^{\circ} \mathrm{C}$, a second weight loss begins, which corresponds to the depolymerisation and degradation into water and gases. ${ }^{15}$ A maximum degradation of weight loss i.e., 85 and $95 \%$ was observed up to $395{ }^{\circ} \mathrm{C}$ for both Glc-BC and Gly-BC respectively.

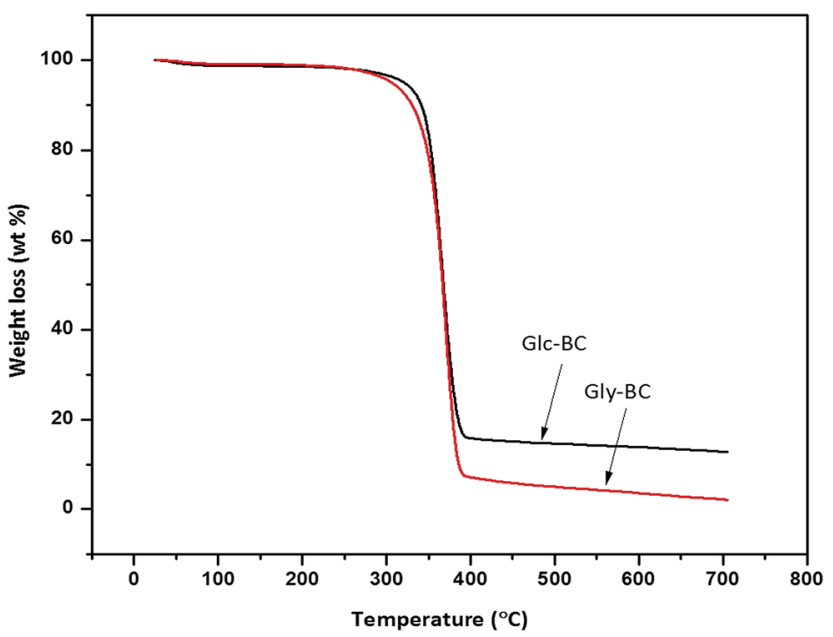

Fig. 9 Thermogravimetric analysis (TGA) curves of Gly-BC and Glc$\mathrm{BC}$.

\section{Conclusions}

Isolation of $K$. rhaeticus was only reported from a fermented beverage; i.e., kombucha tea. For the first time, a potential BC producing strain, $K$. rhaeticus $\mathrm{PG} 2$, was isolated from a rotten pomegranate fruit sample. The BC production efficiency of the strain PG2 was assessed using various sugars and sugar alcohols as the carbon source. Glycerol was found to be the most efficient source of carbon utilized by the $K$. rhaeticus PG2 strain, with highest $\mathrm{BC}$ production of $6.9 \mathrm{~g} \mathrm{~L}^{-1}$, as compared to the other sugars. The highest ever BC production of $8.7 \mathrm{~g} \mathrm{~L}^{-1}$ was with $3 \%$ glycerol as a carbon source. This is the highest yield of BC reported for any $K$. rhaeticus strain to date. In a comparative analysis of the $\mathrm{BC}$ produced by glycerol (Gly-BC) and glucose (Glu-BC), Gly$\mathrm{BC}$ has a more compact and less porous structure. The compact packing of the nanofibrils resulted in a smooth surface, which can support the growth and proliferation of cells. The BC obtained from the glycerol source could be used as a tissue scaffold material. Structural analysis revealed that Gly-BC is much more crystalline than Glc-BC. Overall, glycerol is the major by-product of the biodiesel industry, and due to its higher availability, the market price of crude glycerol has dropped down. Our strain, the $K$. rhaeticus PG2, utilizes glycerol very effectively and produces a high yield of $\mathrm{BC}$ with good structural characteristics. The cost effective production of $\mathrm{BC}$ with glycerol and crude glycerol is possible by using an indigenous strain of $K$. rhaeticus PG2.

\section{Conflicts of interest}

There are no conflicts to declare.

\section{Acknowledgements}

Meghana Thorat is thankful to the University Grant Commission (UGC) for a fellowship. The authors would like to thank DBT for a financial grant (No. BT/PR11160/AAQ/3/674/2014), and the Director of the CSIR-National Chemical Laboratory for his continuous support. 


\section{References}

1 N. Lin and A. Dufresne, Eur. Polym. J., 2014, 59, 302-325.

2 S.-S. Wang, Y.-H. Han, Y.-X. Ye, X.-X. Shi, P. Xiang, D.-L. Chen and M. Li, RSC Adv., 2017, 7, 45145-45155.

3 L. Nielsen, X. Li and L. J. Halverson, Environ. Microbiol., 2011, 13, 1342-1356.

4 P. R. Chawla, I. B. Bajaj, S. a. Survase and R. S. Singhal, Food Technol. Biotechnol., 2009, 47, 107-124.

5 I. Sulaeva, U. Henniges, T. Rosenau and A. Potthast, Biotechnol. Adv., 2015, 33, 1547-1571.

6 A. F. S. Costa, F. C. G. Almeida, G. M. Vinhas and L. A. Sarubbo, Front. Microbiol., 2017, 8, 1-12.

7 H. G. Oliveira Barud, H. D. S. Barud, M. Cavicchioli, T. S. Do Amaral, O. B. De Oliveira Junior, D. M. Santos, A. L. De Oliveira Almeida Petersen, F. Celes, V. M. Borges, C. I. De Oliveira, P. F. De Oliveira, R. A. Furtado, D. C. Tavares and S. J. L. Ribeiro, Carbohydr. Polym., 2015, 128, 41-51.

8 W. Czaja, A. Krystynowicz, S. Bielecki and R. M. Brown, Biomaterials, 2006, 27, 145-151.

9 H. Ullah, F. Wahid, H. A. Santos and T. Khan, Carbohydr. Polym., 2016, 150, 330-352.

10 G. Picheth, C. Pirich, M. Sierakowski, M. Woehl, C. Sakakibara, C. de Souza, A. Martin, R. da Silva and R. de Freitas, Int. J. Biol. Macromol., 2017, 104, 97-106.

11 F. Esa, S. M. Tasirin and N. A. Rahman, Agric. Agric. Sci. Procedia, 2014, 2, 113-119.

12 Z. Shi, Y. Zhang, G. O. Phillips and G. Yang, Food Hydrocolloids, 2014, 35, 539-545.

13 C. Molina-Ramírez, M. Castro, M. Osorio, M. TorresTaborda, B. Gómez, R. Zuluaga, C. Gómez, P. Gañán, O. J. Rojas and C. Castro, Materials, 2017, 10(6), 639.

14 F. Mohammadkazemi, M. Azin and A. Ashori, Carbohydr. Polym., 2015, 117, 518-523.

15 R. T. A. Machado, J. Gutierrez, A. Tercjak, E. Trovatti, F. G. M. Uahib, G. D. P. Moreno, A. P. Nascimento, A. A. Berreta, S. J. L. Ribeiro and H. S. Barud, Carbohydr. Polym., 2016, 152, 841-849.
16 R. A. C. Dos Santos, A. A. Berretta, H. D. S. Barud, S. J. L. Ribeiro, L. N. González-García, T. D. Zucchi, G. H. Goldman and D. M. Riaño-Pachón, Genome Announcements, 2014, 2, e00731.

17 A. Suwanposri, P. Yukphan, Y. Yamada and D. Ochaikul, Maejo Int. J. Sci. Technol., 2013, 7, 70-82.

18 S. Hestrin and M. Schramm, Biochem. J., 1954, 58, 345-352. 19 M. Poletto, H. L. Ornaghi Júnior and A. J. Zattera, Materials, 2014, 7, 6105-6119.

20 C. Zhong, G. C. Zhang, M. Liu, X. T. Zheng, P. P. Han and S. R. Jia, Appl. Microbiol. Biotechnol., 2013, 97, 6189-6199.

21 M. Ishihara, M. Matsunaga, N. Hayashi and V. Tišler, Enzyme Microb. Technol., 2002, 31, 986-991.

22 E. Tsouko, C. Kourmentza, D. Ladakis, N. Kopsahelis, I. Mandala, S. Papanikolaou, F. Paloukis, V. Alves and A. Koutinas, Int. J. Mol. Sci., 2015, 16, 14832-14849.

23 S. M. Keshk, J. Bioprocess. Biotech., 2014, 4, 1-10.

24 P. Semjonovs, M. Ruklisha, L. Paegle, M. Saka, R. Treimane, M. Skute, L. Rozenberga, L. Vikele, M. Sabovics and I. Cleenwerck, Appl. Microbiol. Biotechnol., 2017, 101, 10031012.

25 K. A. Zahan, N. Pa'e and I. I. Muhamad, Arabian J. Sci. Eng., 2015, 40, 1881-1885.

26 A. Vazquez, M. L. Foresti, P. Cerrutti and M. Galvagno, J. Polym. Environ., 2013, 21, 545-554.

27 D. L. VanderHart and R. H. Atalla, Macromolecules, 1984, 17, 1465-1472.

28 C. Castro, R. Zuluaga, J. L. Putaux, G. Caro, I. Mondragon and P. Gañán, Carbohydr. Polym., 2011, 84, 96-102.

29 A. Kumagai, M. Mizuno, N. Kato, K. Nozaki, E. Togawa, S. Yamanaka, K. Okuda, I. M. Saxena and Y. Amano, Biomacromolecules, 2011, 12, 2815-2821.

30 M. S. Dayal, N. Goswami, A. Sahai, V. Jain, G. Mathur and A. Mathur, Carbohydr. Polym., 2013, 94, 12-16.

31 S. Sheykhnazari, T. Tabarsa, A. Ashori, A. Shakeri and M. Golalipour, Carbohydr. Polym., 2011, 86, 1187-1191.

32 B. V. Mohite and S. V. Patil, Carbohydr. Polym., 2014, 106, 132-141. 